\title{
Comparing Compartment Fires with Compartment Fire Models
}

\author{
HAROLD E. NELSON and SCOT DEAL \\ Building and Fire Research Laboratory \\ National Institute of Standards and Technology \\ Washington, DC 20899, USA
}

\begin{abstract}
An approach for appraising the expected performance of compartment fire models is presented. The approach involves comparing the results of well documented test data to selected outputs of the model. The paper applies this approach to four zone compartment fire models and offers a brief analysis of the results of that application. The test data was obtained from room fire tests involving both wood and plastic cribs reported by Quintiere and McCaffery in 1980. The models compared were FIRST9X, FAST, CCFM-VENTS, AND FPETOOL.
\end{abstract}

\section{OBJECTIVE}

This paper suggests an approach to assist users of compartment fire models in appraising the degree of trust to be placed in a particular compartment fire model. The selected approach is then used to appraise the performance of four separate models that differ widely in sophistication and user requirements.

\section{METHOD}

The proposed method involves the following elements.

1. Examination (to reasonable understanding) of the basic principles involved and approaches used by the model being investigated. It is important that users understand that models are but instruments which apply selected equations and relationships to the solution of problems. The user, by running the model, tacitly agrees that the selected set of procedures are appropriate for the problem at hand.

2. Choosing a set of model output results that demonstrate the effectiveness of the model being evaluated. Emphasis being placed on the areas of interest to the user. These may vary from case to case. For example, in this presentation, the four variables of upper 
layer temperature, interface level, upper layer oxygen content, and the compartment vent outflow have been selected. These variables test aspects of the model's ability to apply plume dynamics and to conserve mass, momentum, and species. In addition, these variables are normally important parameters in fire safety decisions.

3. Selection of a well characterized and measured test series having input and output data comparable to the model of concern. The choice of the test is important. While many test series have been run, relatively few have the comprehensive and careful measurements necessary to evaluate a model. For example, well instrumented tests often fail to provide information on the burning rates of the materials used for the source fire. Others concentrate on single results such as temperature.

4. Execution of fire simulations using the model being evaluated to produce the results of interest. Careful attention must be given to describing the input parameters accurately. In some cases, the users may choose to undertake sensitivity studies by varying parameters. Such variation, however, should not be used as a method of forcing the model results to fit the experimental data.

5. Comparison of results. In comparing results, the user must understand that the reality of accidental fire is more varied and complex than can be exactly described by any existing collection of equations or exactly measured in any test. Some deviation should always be expected. The deviation from reality may occur in the predictions by the model, in the measurements undertaken in the tests, or most likely, a combination of both.

\section{EXAMPLE APPLICATION OF METHOD}

The suggested method has been test applied to four zone compartment fire models. These models were compared to four tests selected from the work of Quintiere and McCaffrey [1]. The model selection was based on the availability of the models and the depth of understanding of these models by the authors. The data set choice was based on the excellence and scope of the data collected.

The models chosen varied widely in degree of sophistication and user requirements. The models used were:

1. FIRST9X [2]. This is the most recent version of the one-room HARVARD code, also known as HARVARD V. It is also the most detailed model both in terms of user input requirements and model output. It is intended for the sophisticated user who understands and desires alternative routines for modeling specific fire phenomena. For instance the procedures or variables governing plume dynamics, radiative participation of hot layer gases and convective wall heat fluxes are selected by the user. The model output includes details of the radiative heating component from the flames or the conductive heat loss through the walls. FIRST9X is the only model of the four examined that considers more than one fuel object or is capable of enhancing burning rate due to radiative feedback from the enclosure.

2. FAST [3]. FAST is the core model of the HAZARD I [4] package. In this exercise, it was used as a single compartment model, however, it is capable of multiple room analysis. FAST like FIRST9X couples the conservation equations in its fire physics routines. Its treatment of convective wall heat loss is more sophisticated than FIRST9X, but the scope 
of its output is not as broad as FIRST9X. Its input and output are attractively packaged in a menu driven format.

3. CCFM-VENTS [5]. This multi-room model also rigorously applies the conservation equations of mass and momentum to its fluid dynamics. It, however, lacks a mechanism for throttling the fuel's heat release rate due to oxygen starvation. The model also applies a user prescribed value rather than a computational approach to wall and ceiling heat transfer. Its speed relative to FAST, however, makes it an attractive option for multiroom and or forced ventilation studies.

4. FPETOOL [6]. FPETOOL is a collection of equations and models. The main model in FPETOOL is titled FIRE SIMULATOR. FIRE SIMULATOR uses a simple point source plume model. Its fluid dynamics are based on both correlations and conservation equations. In FIRE SIMULATOR the heat release rate is coupled to the availability of oxygen. It uses a series of equations combining simple correlations and physics to predict time variant heat transfer to the room boundaries.

The above list is in descending order of both sophistication required by the user and execution time required by the computer. FPETOOL has the fastest execution time. For the cases used in this study, if a given simulation is executed by FPETOOL in one minute, then the approximate execution times for the other models are: 8,20 and 40 minutes for CCFM VENTS, FAST and FIRST9X, respectively. Users are therefore faced with a choice between level of sophistication and rigor of physics, detail of output, and time investment when choosing which models to apply to a particular problem.

\section{TEST SET USED FOR EXPERIMENTAL DATA}

Four tests were chosen for this study which included two different fuels and two different door widths. Sugar pine was burned in two tests while rigid polyurethane was burned in the other two tests. Both fuels were arranged as cribs. The sugar pine crib fuel packages contained $14 \mathrm{~kg}$ mass whereas the rigid polyurethane crib fuel packages had $9.5 \mathrm{~kg}$ of mass. The vent widths were referred to as full and quarter width. The full width vent was $0.79 \mathrm{~m}$ wide and the quarter width vent was $0.19 \mathrm{~m}$ wide. In all tests the vent height was 1.83 meters. Each material was tested with each vent size. The experimental room measured 2.18 meters on a side and 2.41 meters in height.

Instrumentation included a load cell, thermocouple trees, bidirectional velocity probes, and gas sampling ports. The heat of combustion was determined experimentally using oxygen calorimetry. Visual observations were kept of the interface height.

\section{HEAT RELEASE RATE}

The heat release rate was determined by multiplying the mass loss rate data with an effective heat of combustion. The heat of combustion was determined from standard texts [7] and experimental values [1] while the mass loss rate was determined from the slope of the instantaneous load cell/time curve. Figure 1 shows plots of the calculated heat release rates for each of the four tests. These data were used as the fire input to each of the four models. 


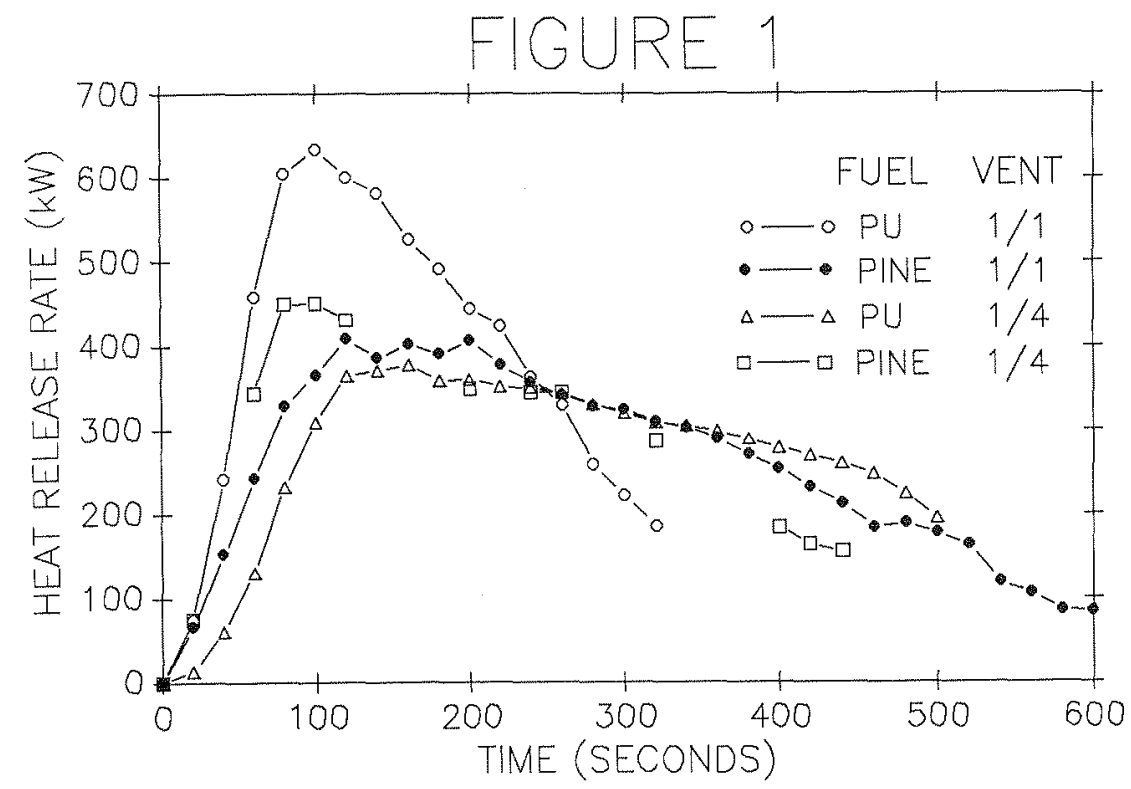

4 PINE CRIBS $1 / 4$ VENT WIDTH time $=300$ SECONDS

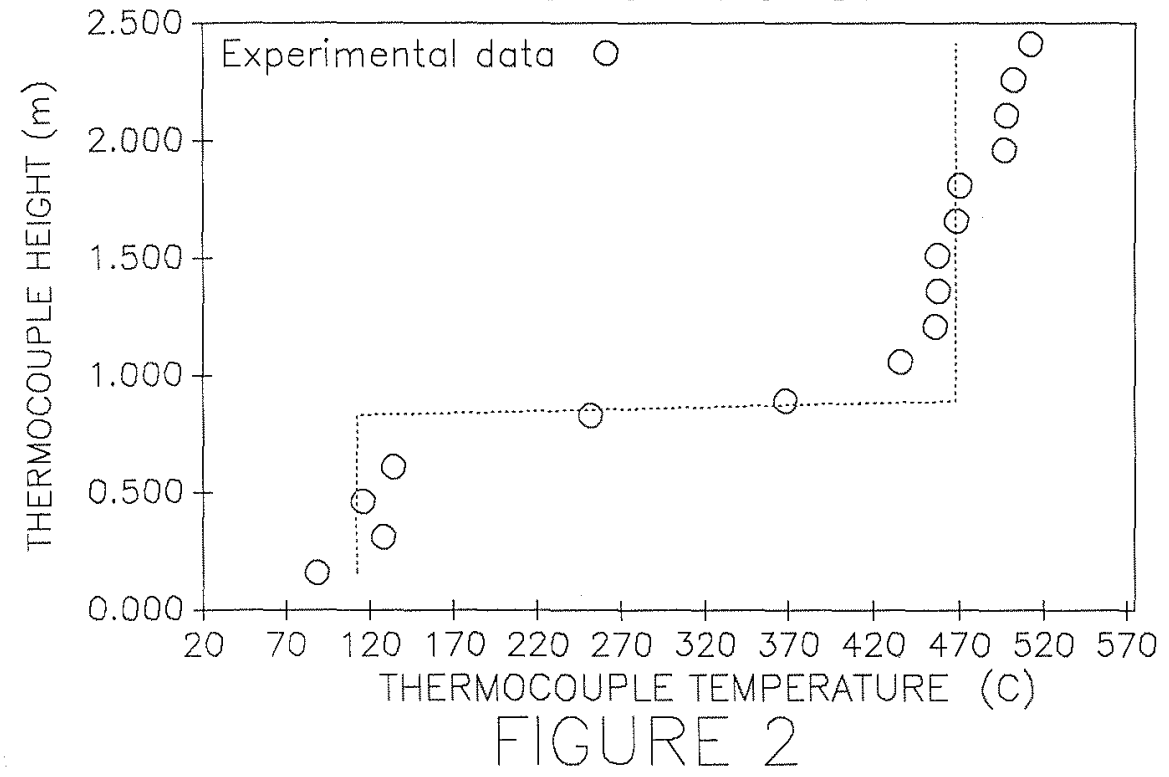




\section{UPPER LAYER TEMPERATURE \& INTERFACE HEIGHT}

Zone fire models simulate the compartment fire environment as a distinct upper and lower layer, each with a uniform temperature throughout its boundaries. The experimenters recorded temperatures at regularly spaced intervals along a thermocouple tree extending from floor to ceiling. To compare the test results with the output of a zone model it is necessary to convert this vertical array of temperature data into equivalent averaged upper and lower layer temperatures and to determine the height of the interface separating these layers.

Mitler's method [8] was chosen for performing the data transformation. The method's mechanics are discussed in his paper. In applying this method the interface height was determined 'a priori' via a freehand determination of the inflection point in the temperature profiles. A typical temperature profile as might be expected in an open door room test and its two zone representation appear in Figure 2.

\section{VENT MASS FLOW RATES}

The vent mass inflow and outflow rates were determined from interpolation of points graphed in Volume II of the test report [1]. These in turn were determined from application of the continuity equation. Density and velocity measurements were taken from a vertical instrument rake located in the center of the doorway. In the tests, data were collected during 35 second traverses of the doorway instrument rake. Both the in and outflowing gases were plotted. The outflow is expected to be the more precise of the two measurements.

\section{OXYGEN CONCENTRATION}

In the test series the only point of oxygen measurement was a sampling tube located in the upper portion of the doorway. The oxygen concentration in the smoke layer was assumed to equal that registered by this gas sampling tube.

\section{RESULTS}

\section{TEMPERATURE}

Figures $3,4,5$, and 6 are composites of four graphs each. These Figures compare the models with each other and with the experimental data. In general, all four of the models gave reasonable approximations of upper layer temperatures. There are some exceptions however. In the wide open vent configurations, the CCFM model using the default values for heat loss significantly under-predicted the actual results. It is believed that this is due to the very large vent versus room size in the test facility. Test runs using CCFM with a reduced portion of the energy loss to the walls brings the temperature prediction in line. Currently, however, the instructions with CCFM do not guide the user in making this adjustment. Such guidance could increase the confidence and useability of CCFM. 

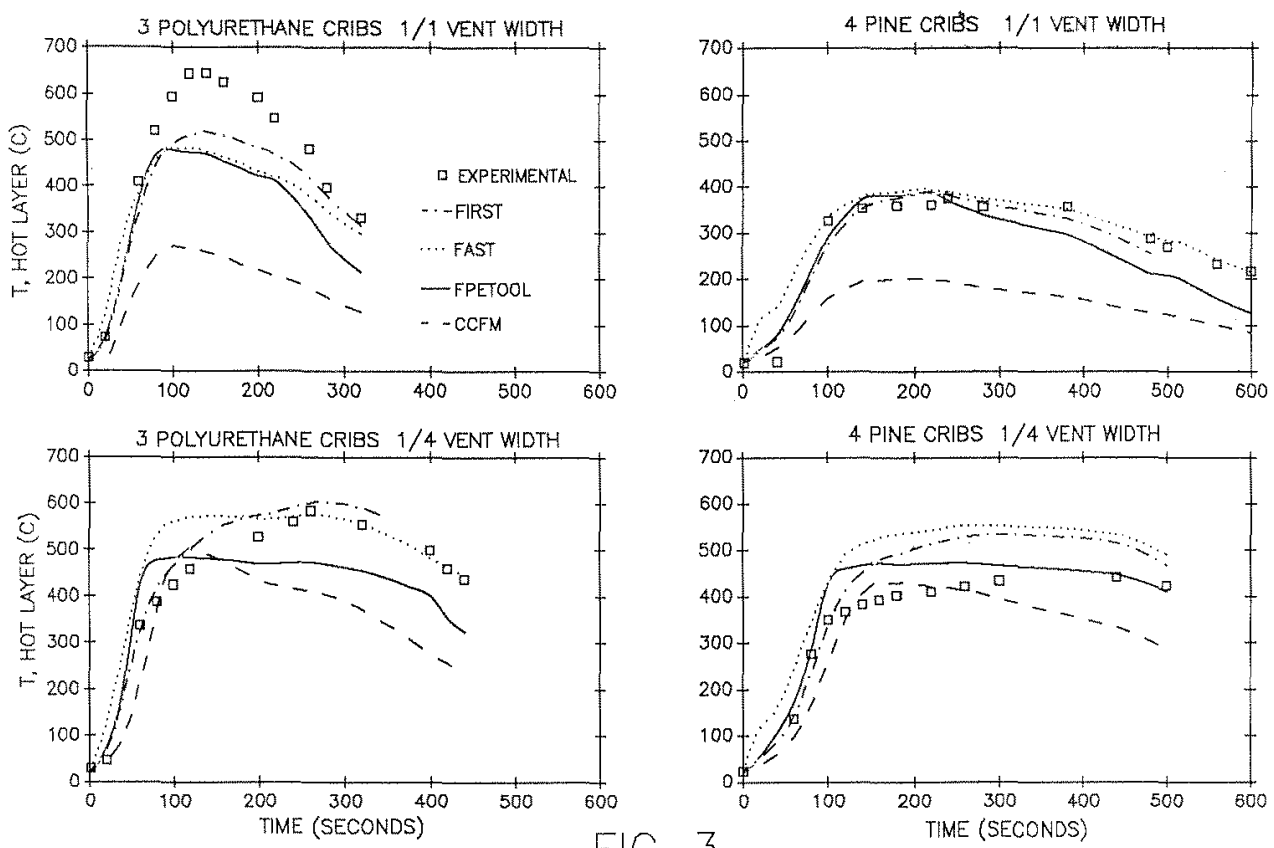

FIG. 3
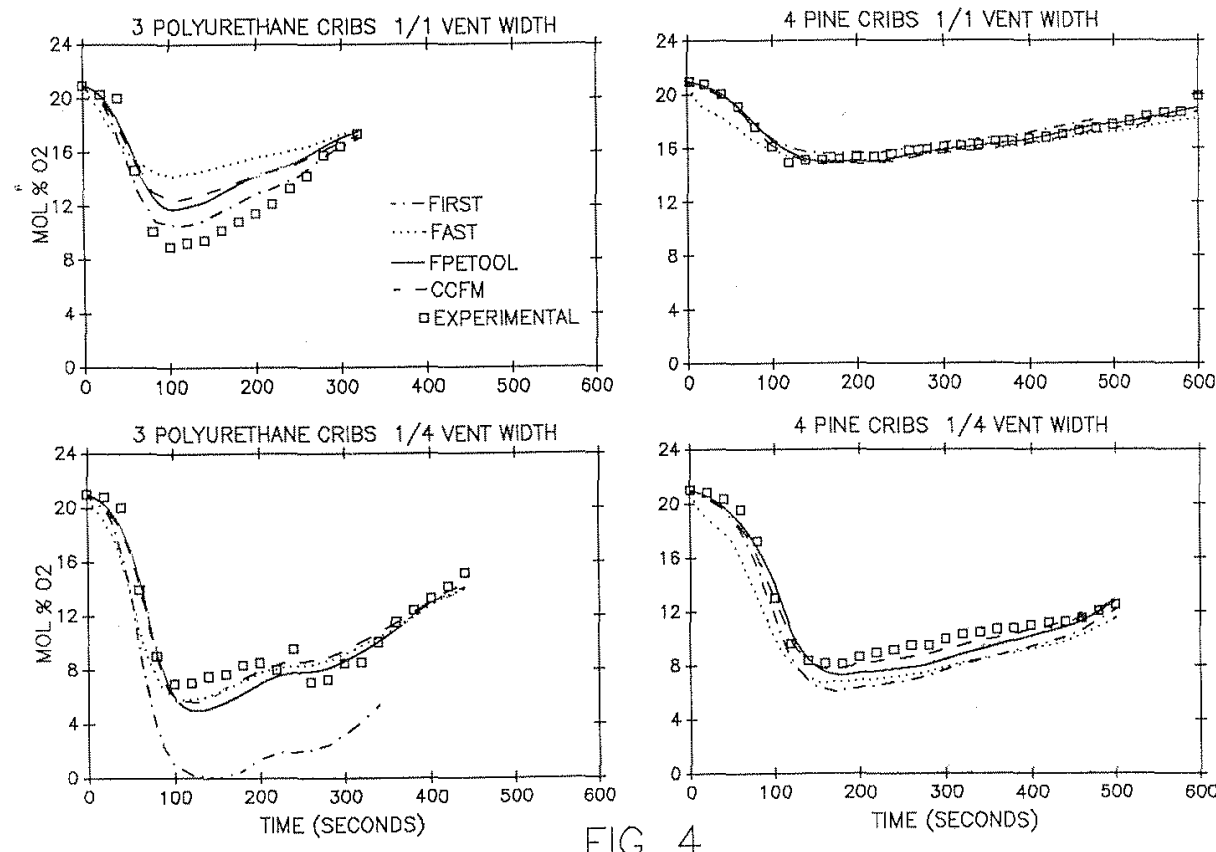

FIG. 4 

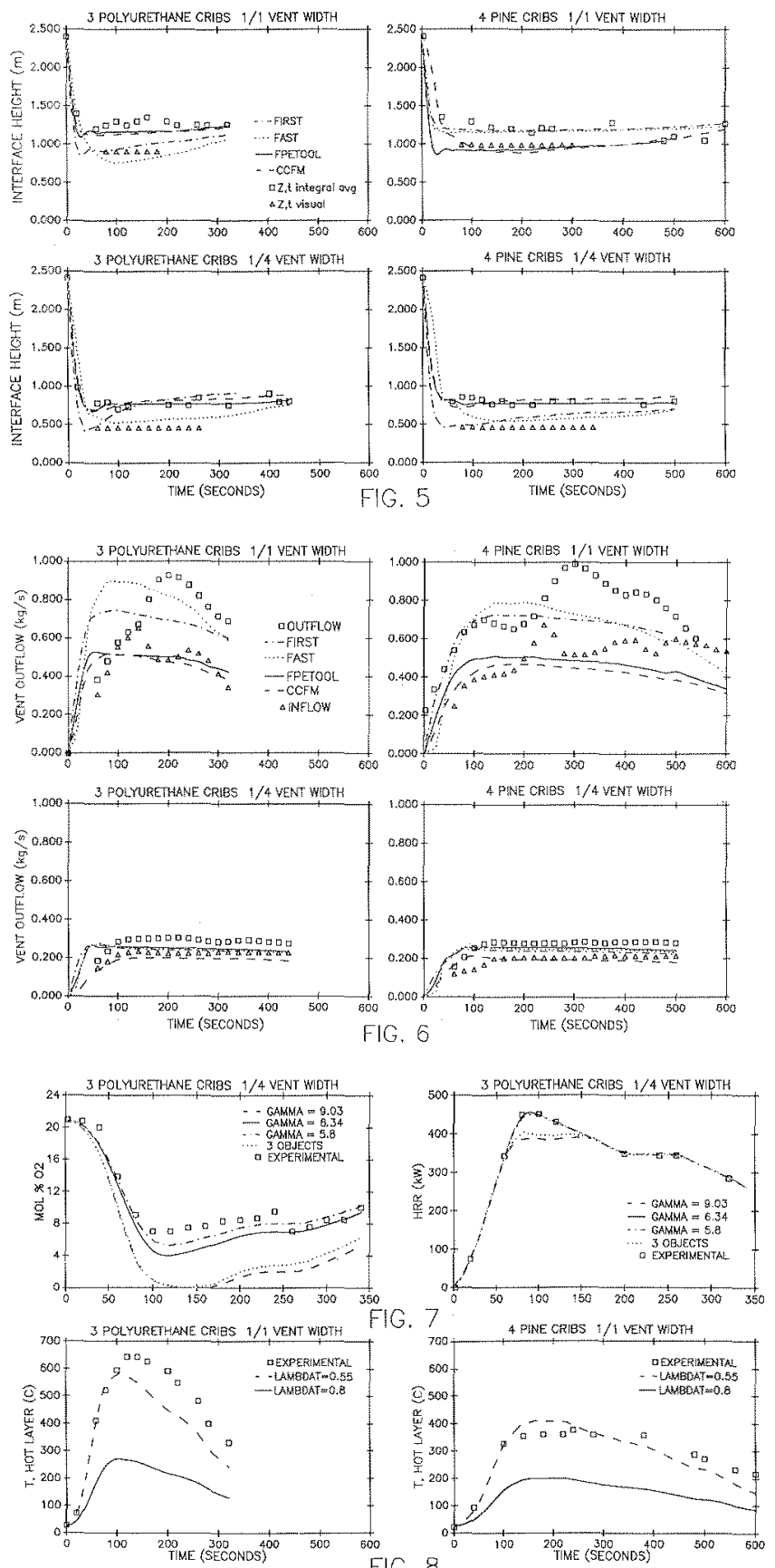
FIG. 8 


\section{OXYGEN CONCENTRATION}

All of the models agreed well among themselves and with the data, with one exception. The FIRST9X model overpredicted the oxygen consumption in the narrow door configuration with polyurethane as the fuel. With respect to fuels, the wood fueled fires were better predicted than the polyurethane fires.

\section{INTERFACE HEIGHT}

All of the models indicated interface heights relatively close to those reported from the test results. This is to be expected as interface heights are the least sensitive and best predicted parameter of the models.

\section{VENT FLOWS}

All of the models predicted vent flows quite well with the narrow vents but scattered widely with the wide vent. At this time, we do not have an explanation for the spread. It is interesting that FPETOOL and CCFM do not use the same flow algorithms though they seem to plot the same values. Since both appear to under estimate the tested vent flow rates, this emphasizes a potential problem of using one model to test another.

\section{SENSITIVITY ANALYSIS}

A sensitivity study was pursued with several input parameters of the FIRST9X and CCFM VENTS models because of apparent anomalies in the oxygen and temperature predictions seen in Figures 3 and 4 . The objective was to identify the parameters responsible for the deviations and rank the sensitivity of these parameters.

The problem the FIRST9X model had in predicting the narrow door/ polyurethane fuel experiment (Figure 4) was that the model perceived that there was a lack of oxygen reaching the fuel. Parameters effecting the entrainment rate were examined as a possible way to have the model increase the amount of oxygen calculated as available to the fuel. These parameters were the number of fuel objects, the entrainment model and the air/fuel mass ratio (labeled as gamma in Figure 7.) The number of fuel objects (and hence plumes) was varied from one to three, the Zukoski [9] and McCaffrey [10] entrainment correlations were compared, and the air/fuel mass ratio was varied from the experimental, stoichiometric and empirical values $(9.03,6.34,5.8)$. The air/fuel mass ratio had the greatest effect. The fuel/air ratio which produced the best fit with the was 5.8. This is, however, only about $60 \%$ of the experimental value. The fact that the experimental air/fuel ratio could not reproduce the heat release rate curve was discouraging (see Figure 8). The stoichiometric ratio produced satisfactory results at both full and narrow vent configurations, however, the stoichiometric ratio ignores the fact that char is formed, an item explicitly accounted for in the experimental ratio. The reasons for these deviations are not apparent to the authors. It appears this is a topic that justifies further exploration since it would be very convenient to model a variety of solid fuels with the detail that is possible with FIRST9X.

CCFM VENTS underpredicted the hot layer temperatures of both full door experiments. An obvious parameter to investigate was the wall heat loss fraction since this is a user stipulated value (LAMT) which is heuristicly determined. The heat loss fraction fixes the portion of the 
heat released from the fire which transfers through the compartment linings (walls, ceiling and floor.) Figure 8 illustrates that a room lining heat loss percentages of 55\% (rather than the $80 \%$ default value) produced much better simulations. The $55 \%$ loss factor also agrees with a value produced by a method very similar to the McCaffrey, Quintiere, Harkleroad [11] of estimating effective heat loss to room linings ${ }^{1}$. Changing the wall heat loss fraction had little effect upon the parameters other than temperature. The reason for the anomaly with this heat loss parameter appears to be vent oriented. The fraction of the enclosure area occupied by the door at the larger vent configurations is roughly three times that of a typical door opening in residential compartments.

The heat of combustion, the height of the fire, and species generation rates were other input parameters which required engineering judgement to ascertain. The heats of combustion were experimentally provided[1] and cross-referenced with literature values[7,12]. Product generation rates were also found in the literature.[7,14]. The fire height was modeled as the level at which entrainment begins or at the base of the flames.

\section{SUMMARY}

The authors were surprised by the apparent ability of all four models tested to provide reasonable approximations of the fire tests used. Since the input heat release rates were derived from test load cell data any problems involved in using free burn data as an input to room fire modeling were not addressed. It is felt that the results indicate a reasonable level of confidence can be placed in these models, at least in one room configurations impacted by fires of the size represented by the test series.

It is also felt that the suggested five step approach provides a rational approach the examination of the expected accuracy of the use of a model in an actual engineering application.

\section{BIBLIOGRAPHY}

1. Quintiere, J.G., McCaffrey, B.J., "The Burning of Wood \& Plastics in an Enclosure--Vol. I\&II," NBSIR 80-2054, National Institute of Standards and Technology (formerly National Bureau of Standards), Gaithersburg, MD, November 1980.

2. Mitler, H.E., Rockett, J.A., "User's Guide to FIRST, A Comprehensive Single-Room Fire Model," NBSIR 87-3595, National Institute of Standards and Technology (formerly National Bureau of Standards), Gaithersburg, MD, 1987.

3. Jones, W.W., Peacock, R.D., Refinement and Experimental Verification of a Model for Fire Growth and Smoke Transport, Proceedings of the Second International Symposium on Fire Safety Science, Tokyo (1988).

4. Bukowski, R.W., Peacock, R.D., Jones, W.W., Forney, C.L., "Technical Reference Guide for the HAZARD I Fire Hazard Assessment Method," NIST Handbook 164, Volume II National Institute of Standards and Technology, Gaithersburg, MD, 1979.Gaithersburg, MD 20899, June 1989.

\footnotetext{
${ }^{1}$ This algorithm is included in FIRE SIMULATOR, the main model in FPETOOL [6]
} 
5. Forney, G.P., Cooper, L.Y., "Plan for the Development of the Generic Framework and Associated Computer Software for a Consolidated Compartment Fire Model Computer Code," NBSIR 86-3500, National Institute of Standards and Technology (formerly National Bureau of Standards), Gaithersburg, MD, January 1987

6. Nelson, H.E., "FPETOOL--Fire Protection Tools for Hazard Estimation," NISTIR 4380, National Institute of Standards and Technology, Gaithersburg, MD, 1990.

7. DiNenno, P.J. et. al. eds., SFPE Handbook of Fire Protection Engineering, NFPA, September 1988.

8. Mitler, H.E., "Zone Modeling of Forced Ventilation Fires," Combustion Science \& Technology, 1984, Vol. 39, pp. 83-106.

9. Zukoski, E. E., Kubota, T. and Cetegen, B.; "Entrainment of Fire Plumes", Fire Safety Journal (3) (1980/81) 107

10. McCaffrey, B. J.; "Purley Buoyant Diffusion Flames: Some Experimental Results", NBSIR 79-1910, National Institute of Standards and Technology (formerly National Bureau of Standards), Gaithersburg, MD, 1979.

11. McCaffrey, B. J., Quintiere, J. G. and Harkleroad, M. F; "Estimating Room Temperatures and the Likelihood of Flashover Using Fire Test Data Correlations", Fire Technology, (17), No. 2 98-117, May 1981.

12. Tewarson, A., "Heat Release Rates From Burning Plastics," Journal of Fire and Flammability, Vol. 8, Jan., 1977, pp. 115-130.

13. Paabo, M., Levin, B., "A Review of the Literature on the Gaseous Products and Toxicity Generated From the Pyrolysis and Combustion of Rigid Polyurethane Foams," NBSIR 85-3224, National Institute of Standards and Technology (formerly National Bureau of Standards), Gaithersburg, MD, 1985.

14. Hume, J., Owen, R., "Smoke Production by Burning FR Flexible PU Foam," 2nd Int'1 Conference on Flame Retardants, Proceedings, London, England, November, 1986, pp 20/1-7.

This paper is a contribution of the National Institute of standards and Technology and is not subject to copyright. 